\title{
SISTEMA DE EVALUACIÓN BASADO EN COMPETENCIAS ORIENTADO HACIA UNA EDUCACIÓN DE CALIDAD Y SU INFLUENCIA EN EL RENDIMIENTO ACADÉMICO EN ALUMNOS INGRESANTES DE LA ESCUELA PROFESIONAL DE MATEMÁTICA DE LA UNIVERSIDAD NACIONAL JORGE BASADRE GROHMANN DE TACNA - 2016
}

\begin{abstract}
COMPETENCY-BASED EVALUATION SYSTEM ORIENTED TOWARDS A QUALITY EDUCATION AND ITS INFLUENCE ON ACADEMIC PERFORMANCE IN STUDENTS IN THE PROFESSIONAL SCHOOL OF MATHEMATICS OF THE NATIONAL JORGE BASADRE GROHMANN DE TACNA UNIVERSITY - 2016
\end{abstract}

\author{
Humberto vargas Pichon, Luis Solorzano Espinolą; Ita Daneri Huaman Guzman ${ }^{3}$
}

\section{RESUMEN}

El presente trabajo de investigación es de tipo cuasi experimental. Su objetivo es determinar en qué medida un sistema de evaluación, basado en competencias orientado hacia una Educación de calidad, influye significativamente en el rendimiento académico en alumnos ingresantes de la Escuela Profesional de Matemática de la Universidad Nacional Jorge Basadre Grohmann de Tacna - 2016.

La población comprendió 32 estudiantes y la muestra conformada por 17 estudiantes cuya selección se consideró teniendo un muestreo por conveniencia

Se concluye que: En aplicación de la encuesta se tiene aceptables niveles de la conducta asertiva y en relación a los niveles del rendimiento académico se encuentra un significativo nivel insatisfactorio en el rendimiento académico de los estudiantes ingresantes del primer año de la Escuela Profesional de Ingeniería Metalúrgica y Materiales, de la Universidad Nacional Jorge Basadre Grohmann- Tacna, 2016. ( $p$-Valor $=0,000<0,05$ para el I Ciclo y $p$-Valor $=0,000<0,05$ para el II Ciclo).

Palabras Clave: Sistema de evaluación y rendimiento académico.

\section{ABSTRACT}

The present work of investigation is of type quasi experimental. Its objective is to determine the extent to which a system of evaluation, based on competences oriented towards a quality education, significantly influences the academic performance in incoming students of the Professional School of Mathematics of the National University Jorge Basadre Grohmann of Tacna - 2016.

The population comprised 32 students and the sample consisted of 17 students whose selection was considered having a sampling for convenience.

There is a significant difference between the average of academic achievement scores of students in the experimental group, after applying a Competency-based Assessment System oriented towards a Quality Education in incoming students of the National University's Mathematics Professional School Jorge Basadre Grohmann of Tacna - 2016 ( $p$-Value $=0.000<0.05$ for I Cycle and p-Value $=0.000$ $<0.05$ for II Cycle).

Key Words: Academic evaluation and performance system.

Keywords: Hyperbolic differential equations, existence and uniqueness of the generalized solution.

\section{INTRODUCCIÓN}

En la actualidad las instituciones de educación superior se encuentran inmersas en procesos profundos de análisis y reflexión debido a las exigencias que enfrentan, por los cambios significativos en su entorno. A diferencia de épocas anteriores, el conocimiento en sus distintos saberes se ha transformado en un factor decisivo para la inserción a un mundo globalizado y a un mercado internacional, en donde el intercambio de capital humano, de bienes materiales y bienes culturales, se ha

\footnotetext{
1 Doctor en Administración de la Educación

Docente de la Facultad de Ciencias - Universidad Nacional Jorge Basadre Grohmann - Tacna Perú

2 Msc. con mención en Tecnología Educativa

Docente de la Facultad de Ciencias - Universidad Nacional Jorge Basadre Grohmann - Tacna Perú

3 Licenciada en Obstetricia

Docente de la Facultad de Ciencias Jurídicas y Empresariales - Universidad Nacional Jorge Basadre Grohmann - Tacna Perú 
convertido en una necesidad primordial de la sociedad actual. Frente a esta revolución económica, política y sociocultural, los centros de educación superior se plantean procesos de grandes transformaciones en sus sistemas académicos, particularmente en sus estructuras curriculares que requieren de un alto grado de articulación y concreción para poder procesar los constantes cambios.

\section{El espacio europeo de educación superior}

Con las Declaraciones de Sorbona (1998), y Bolonia (1999), se marcó el inicio del proceso de concertación para la transformación de la educación superior más trascendental del siglo XX en la mayoría de los países del continente europeo. Esta propuesta más conocida como "el Proceso de Bolonia" o "Convergencia Europea", procuraba para el año 2010, haber conformado el Espacio Europeo de Educación Superior (EEES). Así, se desarrolló el Proyecto Tuning Educational Structures in Europe (PTESE); una iniciativa de las universidades con el fin de encontrar conjuntamente puntos comunes de referencia, que facilitarán la libre circulación internacional de los estudiantes y profesores, en estos centros de educación superior tal como lo declara el Proceso de Convergencia

Para el desarrollo del Proyecto Tuning, se tomó como marco de referencia las experiencias acumuladas de los programas ERASMUSSOCRATES, especialmente en lo que respecta al Sistema Europeo de Transferencia y Acumulación de Créditos (ECTS). Para las instituciones de educación superior, el PTESE, fue un punto de partida para la homologación de las estructuras curriculares y contenidos de estudio que la colectividad académica donde más de 100 expertos europeos, definieron en ese espacio de diálogo y reflexión, los posibles perfiles educativos y profesionales que la sociedad exige y el nivel de formación que los estudiantes en términos de competencias y resultados de aprendizaje deberían alcanzar.

EI PTESE, definió el término Competencia como "Una combinación dinámica de atributos, en relación a procedimientos, habilidades, actitudes y responsabilidades, que describen los encargados del aprendizaje de un programa educativo o lo que los alumnos son capaces de demostrar al final de un proceso educativo" (Bravo, 2007, p. 13). En tal sentido, los expertos académicos organizaron las competencias en un conjunto de conocimientos, habilidades, destrezas y actitudes, las cuales se espera que los estudiantes adquieran y dominen durante, o al término de un ciclo de estudio, proceso corto o largo de aprendizaje, igualmente, identificadas con programas completos de estudio o con unidades individuales de aprendizaje.

\section{Contexto latinoamericano}

Pese a las marcadas diferencias que le caracterizan, América Latina, no podría mantenerse al margen de los vertiginosos cambios y negarse la oportunidad de encontrar un lugar más equitativo y justo, tanto en la economía regional como en el mercado mundial. La transformación de la educación superior en Latinoamérica, estaba sujeta a una serie de factores o demandas que deben tomarse en cuenta al momento de impulsar las reformas, si se espera obtener cambios genuinos, significativos y perecederos. Ello es, que promuevan el desarrollo socio-económico de los países, y que ofrezcan oportunidades en la mejora de la calidad de vida de sus ciudadanos. Así, la UNESCO, a partir de la década de los 90 llevó a cabo un proceso de consulta con diferentes actores de la sociedad tanto a nivel mundial como a nivel regional, que le permita elaborar las políticas educativas de la educación superior. Tal fue el caso de la reunión internacional de reflexión "Los nuevos roles de la educación superior en América Latina" sostenida en Caracas, Venezuela en mayo de 1991, y organizada por el Centro Regional para la Educación en América Latina y el Caribe (CRESALC).

Consecuentemente, se llevó a cabo la Conferencia Regional sobre Políticas y Estrategias para la Transformación de la Educación Superior en América Latina en la República de Cuba en noviembre de 1996 con 26 países participantes. En este espacio de reflexión, se presentaron propuestas concretas respecto a los principios fundamentales que, a juicio de los participantes, eran los necesarios para impulsar la reforma. Igualmente, se elaboró un plan de acción que condujera progresivamente, al desarrollo de la educación permanente y sin fronteras, en un nuevo contexto de apoyo y cooperación nacional e internacional para tal efecto.

En el marco de reflexión sobre la necesidad de transformación de la educación superior, y en el espacio de colaboración de la Comisión Europea a través del programa ALFA, surge el proyecto Tuning-América Latina; "trabajo conjunto que busca y construye lenguajes y mecanismos para 
la comprensión recíproca de los sistemas de enseñanza superior, que faciliten los procesos de reconocimiento de carácter transnacional y regional. El proyecto Tuning-América Latina se desarrolló con la participación de más de 200 académicos de 19 países de la región, con el propósito de rescatar el papel de la educación superior como centro del pensamiento, del debate, la cultura y de la innovación. Uno de los aportes más significativos del proyecto fue la introducción de la metodología para la comprensión y comparación del currículo introduciendo los conceptos: resultados de aprendizaje y competencias. El primer concepto se define como "el conjunto de competencias que incluye conocimientos, comprensión y habilidades y que se espera que el estudiante domine, comprenda y demuestre después de completar un proceso corto o largo de aprendizaje" (Bravo, 2007 p. 3). Las competencias por otra parte, han sido categorizadas en dos grandes grupos: competencias genéricas, que en principio son independientes del área de estudio $y$ competencias específicas; propias de cada disciplina del conocimiento.

\section{Contexto peruano}

Podemos hacer mención al "I Encuentro Internacional Universitario EL CURRÍCULO POR COMPETENCIAS EN LA EDUCACIóN SUPERIOR: Ponencias y debate", organizado los días 23 y 24 de octubre del 2014 y llevado a cabo en la Pontificia Universidad Católica del Perú. En dicho evento uno de los temas tratados fue: La evaluación por competencias. Se llegó a las siguientes conclusiones:

1. Acerca de la evaluación a nivel de sistema, o macro curricular: Autoevaluación para la acreditación. Condiciones para la implementación: Capacitación docente para que todos comprendan la lógica y proceso del cambio, y Evaluación del currículo.

2. Aspectos que se debe tener en cuenta en el desempeño del docente universitario:

Competencias del perfil del docente en la educación superior (Temuco): - Planificación de la enseñanza (probidad). - Estrategias de enseñanza (tríada tecnología aplicada a la disciplina carrera, asistente y profesor). - Cultura de evaluación. - Comunicación. - Reflexión sobre la propia práctica. - Uso de TIC.
Evaluación docente: - Tipo $360^{\circ}$ (autoevaluación, evaluación del jefe del departamento y del decano, evaluación de colegas y evaluación de estudiantes). - Módulos de capacitación o desarrollo docente.

Taxonomía de Guskey (2002): - Satisfacción o reacciones del docente. - Cambio en las concepciones (aceptación del cambio o hacerlo propio). - Cambio en la práctica (habilidades, acciones, etc.). - Cambio de cultura organizacional. - Cambio en el aprendizaje de los estudiantes.

3. Aspectos por considerar en la evaluación a nivel del aula: - PAS: Persona en Acción en Situación. - Rúbricas: Deben ser lo más analíticas posible; puntuación por niveles excluyentes (se sugiere evitar puntuaciones intermedias). - Poner en la malla la evaluación de acuerdo con el nivel del curso: Cuadro de competencias genéricas, en el que se debe explicitar en qué cursos y cómo se dan; solo ocurren en situaciones de desempeño complejo. - Articulación progresiva de los cursos y, por lo tanto, de las evaluaciones. - Las estrategias de evaluación deben asemejar el escenario real de desempeño.

\section{Contexto Local}

La Escuela Profesional de Matemática de la UNJBG, cuenta con un modelo curricular cuya estructura comparte componentes comunes para todas las Escuelas y componentes específicos de cada Escuela. Este modelo responde al enfoque de competencias académicoprofesionales, asumiendo específicamente los preceptos teóricos de la corriente constructivista o integrativa y la tipología planteada en el marco del Proyecto TUNING Latinoamérica. En este sentido, el Plan recoge, por una parte, las tendencias curriculares actuales, y por la otra, las innovaciones específicas de la disciplina de Matemática, ofreciendo así el acceso al material y formación relevante para que los docentes lleven a cabo una enseñanza dinámica y eficaz, y desarrollen en sus estudiantes un aprendizaje significativo. Se espera que después de 05 años de estudios, distribuidos en 10 semestres académicos de 17 semanas, y en modalidad presencial, sus egresados serán profesionales capacitados para investigar, abordar y dar solución matemática a problemas científicos y tecnológicos utilizando métodos matemáticos modernos. Asimismo, su capacitación les permitirá transmitir y difundir, mediante la 
enseñanza, los conocimientos matemáticos y su utilidad; y contribuye a forjar una sociedad más humana, más justa y más solidaria, manteniendo el respeto y la diversidad cultural y desarrollará competencias: generales, académicas y profesionales.

\section{MATERIALES Y MÉTODO}

Enfoque metodológico: La investigación centró el desarrollo de su metodología dentro del paradigma positivista, bajo un enfoque cuantitativo, tipo básico, diseño cuasi experimental.

Variables de estudio: Sistema de evaluación basado en competencias y rendimiento académico.

La población comprendió 32 estudiantes ingresantes de la Escuela Profesional de Matemática correspondiente al año académico 2016. La muestra estuvo conformada por 17 estudiantes, miembros de la población antes mencionada cuya selección fue usando un muestreo por conveniencia.

Instrumentos de recolección de datos: Se utilizó como técnica la observación y como instrumentos lo manifestado en los sílabos.

Validación de instrumentos: Según la información manifestada en los sílabos de las asignaturas

Validación de las escalas de valores utilizadas: Según criterio de los investigadores.

Análisis estadístico y presentación de los datos: Se hizo usando el la prueba T-Student con un nivel de significancia de $\sigma=0,05$ y con una confiabilidad del 95\% (Prueba de hipótesis por diferencias de medias).

\begin{tabular}{|c|c|c|c|c|c|c|}
\hline \multicolumn{7}{|c|}{$\begin{array}{l}\text { Estadísticos descriptivos de la asignatura: } \\
\text { GEOMETRÍA ANALÍTICA (I Ciclo) }\end{array}$} \\
\hline \multicolumn{7}{|c|}{\begin{tabular}{l|l|l|l|l|l} 
& Míni & Máxim & Medi & Desviaci \\
mo & o & a & estándar naria
\end{tabular}} \\
\hline $\begin{array}{l}\text { PROMED } \\
\text { IO FINAL }\end{array}$ & 17 &, 0 & 17,0 & $\begin{array}{l}8,70 \\
6\end{array}$ & 4,8832 & $\begin{array}{l}23,84 \\
6\end{array}$ \\
\hline \multicolumn{7}{|c|}{ 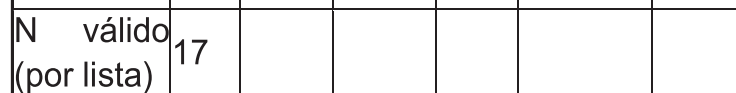 } \\
\hline
\end{tabular}

\section{RESULTADOS}

Estadísticos descriptivos de la asignatura: COMPLEMENTO DE MATEMÁTICAS (I Ciclo)

\begin{tabular}{|l|l|l|l|l|l|l|}
\hline & & Míni & $\begin{array}{l}\text { Máxi } \\
\text { mo }\end{array}$ & $\begin{array}{l}\text { Medi } \\
\text { mo }\end{array}$ & $\begin{array}{l}\text { Desviac } \\
\text { estánda } \\
\text { r }\end{array}$ & $\begin{array}{l}\text { Varia } \\
\text { nza }\end{array}$ \\
\hline $\begin{array}{l}\text { PROMEDI } \\
\text { O FINAL }\end{array}$ & 17 & 10,5 & 16,8 & $\begin{array}{l}13,55 \\
6\end{array}$ & 1,7966 & 3,228 \\
\hline $\begin{array}{l}\text { N válido } \\
\text { (por lista) }\end{array}$ & 17 & & & & & \\
\hline
\end{tabular}

Fuente: Matriz de sistematización de datos, diciembre 2016

\section{Prueba de normalidad}

Prueba de Kolmogorov -Smirnov para una muestra

\begin{tabular}{|c|c|c|c|}
\hline & $\begin{array}{l}\text { PROMEDIO } \\
\text { Geometría } \\
\text { Analítica }\end{array}$ & $\begin{array}{c}\text { PROMEDIO } \\
\text { Complemento } \\
\text { De } \\
\text { Matemáticas }\end{array}$ \\
\hline \multicolumn{2}{|l|}{$\mathrm{N}$} & 17 & 17 \\
\hline \multirow{2}{*}{$\begin{array}{l}\text { Parámetros } \\
\text { normales }^{a, b}\end{array}$} & Media & 8,706 & 13,556 \\
\hline & $\begin{array}{l}\text { Desviación } \\
\text { estándar }\end{array}$ & 4,8832 & 1,7966 \\
\hline \multirow{3}{*}{$\begin{array}{l}\text { Máximas } \\
\text { diferencias } \\
\text { extremas }\end{array}$} & Absoluta & ,128 &, 097 \\
\hline & Positivo &, 123 &, 097 \\
\hline & Negativo &,- 128 &,- 082 \\
\hline \multicolumn{2}{|c|}{ Estadístico de prueba } &, 128 &, 097 \\
\hline \multicolumn{2}{|c|}{$\begin{array}{l}\text { Sig. asintótica } \\
\text { (bilateral) }\end{array}$} &, $200^{c, d}$ &, $200^{c, d}$ \\
\hline
\end{tabular}

a. La distribución de prueba es normal.

b. Se calcula a partir de datos.

c. Corrección de significación de Lilliefors.

d. Esto es un límite inferior de la significación verdadera. 
Estadísticos descriptivos de la asignatura: ESTADÍSTICA DESCRIPTIVA (II Ciclo)

\begin{tabular}{|l|l|l|l|l|l|l|}
\hline & N & Mínimo & Máximo & Media & $\begin{array}{l}\text { Desviación } \\
\text { estándar }\end{array}$ & Varianza \\
\hline $\begin{array}{l}\text { PROMEDIO } \\
\text { FINAL }\end{array}$ & 17 & 1,0 & 14,5 & 9,098 & 4,4343 & 19,663 \\
\hline $\begin{array}{l}\text { N válido (por } \\
\text { lista) }\end{array}$ & 17 & & & & & \\
\hline
\end{tabular}

Fuente: Matriz de sistematización de datos, diciembre 2016

Estadísticos descriptivos de la asignatura: COMPUTACIÓN MATEMÁTICA I (II Ciclo)

\begin{tabular}{|l|l|l|l|l|l|l|}
\hline & N & Mínimo & Máximo & Media & $\begin{array}{l}\text { Desviación } \\
\text { estándar }\end{array}$ & Varianza \\
\hline $\begin{array}{l}\text { PROMEDIO } \\
\text { FINAL }\end{array}$ & 17 & 10,6 & 19,0 & 13,609 & 2,6319 & 6,927 \\
\hline $\begin{array}{l}\text { N válido (por } \\
\text { lista) }\end{array}$ & 17 & & & & & \\
\hline
\end{tabular}

Fuente: Matriz de sistematización de datos, diciembre 2016 Prueba de normalidad

Fuente: Matriz de sistematización de datos, diciembre 2016

Verificación de la hipótesis general para el I CICLO

Prueba de muestras emparejadas

\begin{tabular}{|c|c|c|c|c|c|c|c|c|}
\hline & \multicolumn{5}{|c|}{ Diferencias emparejadas } & & & \multirow[t]{3}{*}{$\begin{array}{l}\text { Sig. } \\
\text { (bilateral) }\end{array}$} \\
\hline & \multirow[b]{2}{*}{ Media } & \multirow{2}{*}{$\begin{array}{l}\text { Desviación } \\
\text { estándar }\end{array}$} & \multirow{2}{*}{$\begin{array}{l}\text { Media de } \\
\text { error } \\
\text { estándar }\end{array}$} & \multicolumn{2}{|c|}{$\begin{array}{l}95 \% \text { de intervalo } \\
\text { de confianza de } \\
\text { la diferencia }\end{array}$} & & & \\
\hline & & & & Inferior & $r$ Superior & $\mathrm{t}$ & $g \mathrm{gl}$ & \\
\hline $\begin{array}{ll}\text { Par } & \text { PROMEDIO_CM } \\
1 & \text { PROMEDIO_GA }\end{array}$ & 5,0000 & 3,8918 &, 9730 & 2,9262 & 7,0738 & 5,139 & 915 &, 000 \\
\hline
\end{tabular}

Prueba de muestras emparejadas

\begin{tabular}{|c|c|c|c|c|c|c|c|c|}
\hline & \multicolumn{5}{|c|}{ Diferencias emparejadas } & & \multirow[b]{3}{*}{$\mathrm{gl}$} & \multirow{3}{*}{$\begin{array}{l}\text { Sig. } \\
\text { (bilateral) }\end{array}$} \\
\hline & \multirow[b]{2}{*}{ Media } & \multirow{2}{*}{$\begin{array}{l}\text { Desviación } \\
\text { estándar }\end{array}$} & \multirow{2}{*}{$\begin{array}{l}\text { Media de } \\
\text { error } \\
\text { estándar }\end{array}$} & \multicolumn{2}{|c|}{$\begin{array}{l}\text { 95\% de intervalo } \\
\text { de confianza de la } \\
\text { diferencia }\end{array}$} & & & \\
\hline & & & & Inferior & Superior & $\mathrm{t}$ & & \\
\hline $\begin{array}{l}\text { Par PROMEDIO_CMI } \\
1 \text { PROMEDIO_ED }\end{array}$ & 4,5108 & 3,0875 & ,7488 & 2,9233 & 6,0982 & 6,024 & 16 &, 000 \\
\hline
\end{tabular}

\section{Verificación de las hipótesis}

\section{Hipótesis específicas}

a) La media de calificaciones en rendimiento académico del Grupo experimental, sin aplicar el Sistema de evaluación basado en competencias orientado hacia una Educación de calidad en alumnos ingresantes de la Escuela Profesional de Matemática de la Universidad Nacional Jorge Basadre Grohmann de Tacna - 2016 es baja. La cual se verifica al tener la media de 8,706 para la asignatura de Geometría Analítica (I Ciclo) y la media de 9,098 para la asignatura de Estadística Descriptiva (II Ciclo). 
b) La media de calificaciones en rendimiento académico del grupo experimental, después de aplicar el sistema de evaluación basado en competencias orientado hacia una educación de calidad en alumnos ingresantes de la Escuela Profesional de Matemática de la Universidad Nacional Jorge Basadre Grohmann de Tacna 2016, es alta. La cual se verifica al tener la media de 13,556 para la asignatura de Complemento de Matemáticas (I Ciclo) y la media de 13,609 para la asignatura de Computación Matemática I (II Ciclo).

\section{Hipótesis general}

Existe una diferencia significativa entre la media de calificaciones en rendimiento académico de alumnos del grupo experimental, después de aplicar un sistema de evaluación basado en competencias orientado hacia una educación de calidad en alumnos ingresantes de la Escuela Profesional de Matemática de la Universidad Nacional Jorge Basadre Grohmann de Tacna 2016.

\section{CONCLUSIÓN}

Debido a que el $p$-valor es 0,000 entonces existe una diferencia significativa entre la media de calificaciones en rendimiento académico de alumnos del Grupo experimental, después de aplicar un sistema de evaluación basado en competencias orientado hacia una educación de calidad en alumnos ingresantes de la Escuela Profesional de Matemática de la Universidad Nacional Jorge Basadre Grohmann de Tacna 2016 correspondientes al II ciclo (Asignaturas de Computación Matemática I: CMI y Estadística Descriptiva: ED).

\section{DISCUSIÓN}

Respecto a los promedios de calificación en I ciclo en las asignaturas de Geometría Analítica, fue de 8,706 con desviación estándar 4,8832, y, Complemento de Matemáticas, fue de 13,556 con desviación estándar de 1,7966. Asimismo, los promedios de calificación en II ciclo en las asignaturas de Estadística Descriptiva, fue de 9,098 con desviación estándar 4,4343, y, Computación Matemática II, fue de 13,609 con desviación estándar de 2,6319, es apoyado por la investigación de Lorenzana (2012), cuando concluye que: Existe un sistema de evaluación de los aprendizajes en donde se integren los referentes teóricos-metodológicos propios del enfoque curricular basado en competencias, se convierte en una herramienta indispensable para orientar de manera correcta y oportuna el quehacer educativo. Así como también concordamos en que hay: La evaluación de los aprendizajes basada en competencias, se identifica como un factor fundamental en la promoción de aprendizajes significativos y perecederos a lo largo de todo el proceso de formación educativa.

Por otro lado, concordamos con la investigación de Sanabria (2003), cuando llega a la conclusión siguiente: Los estudiantes que participaron en el método didáctico de seminario (equivalente con el método trabajo en equipo en la presente investigación) alcanzaron un índice alto, mientras que con la clase magistral (método tradicional) un índice académico bajo.

Asimismo, coincidimos con la investigación de Vildoso (2003), cuando llegó a conclusión siguiente: Existe una correlación significativa entre los hábitos de estudio (entre ellos el método, trabajo en equipo en la presente investigación), la autoestima y el rendimiento académico de los alumnos de segundo, tercero y cuarto año de la Escuela Académico Profesional de Agronomía.

\section{CONCLUSIONES}

En alumnos ingresantes de la Escuela Profesional de Matemática de la Universidad Nacional Jorge Basadre Grohmann de Tacna 2016, la media de calificaciones en rendimiento académico SIN aplicar el sistema de evaluación basado en competencias es baja. Detectado, después de analizar descriptivamente por una media de 8,706 en la Asignatura de Geometría Analítica (I Ciclo) y una media de 9,098 en la Asignatura de Estadística Descriptiva (II Ciclo).

En alumnos ingresantes de la Escuela Profesional de Matemática de la Universidad Nacional Jorge Basadre Grohmann de Tacna 2016, la media de calificaciones en rendimiento académico APLICANDO el sistema de evaluación basado en competencias es alta. 
Detectado, después de analizar descriptivamente por una media de 13,556 en la Asignatura de Complemento de Matemáticas (I Ciclo) y una media de 13,609 en la Asignatura de Computación Matemática I (II Ciclo).

Existe una diferencia significativa entre la media de calificaciones en rendimiento académico, sin aplicar y después de aplicar el sistema de evaluación baso en competencias en alumnos ingresantes de la Escuela Profesional de Matemática de la Universidad Nacional Jorge Basadre Grohmann de Tacna - 2016. Detectado, después de aplicar la prueba T para diferencia de medias, por un $\mathrm{P}-\mathrm{Valor}=0,000$ en el I ciclo y un $\mathrm{P}$-Valor $=0,000$ en el $\mathrm{II}$ ciclo los cuales son menores que 0,05

\section{REFERENCIAS BIBLIOGRÁFICAS}

CABRERA, P. \& GALÁN, E. (2002). Satisfacción escolar y rendimiento académico. Revista de Psicodidáctica. Universidad del País Vasco/Euskal Herriko Unibertsitatea. Vitoria-Gazteis, España, 14, 87-97.

CARRILLO, M. (2015). "El currículo por competencias en la Educación Superior”. Lima: Nova Print S.A.C. Recuperado de: http://vicerrectorado.pucp.edu.pe/academico/ wp-ontent/uploads/2015/03/

curriculo_competencias.pdf

[Consulta 04 de enero del 2017]

CLERICI, C.; MONTEVERDE, A. \& FERNÁNDEZ,

A. (2015) Lectura, escritura y rendimiento académico en ingresantes universitarios. Ciencia, Docencia y Tecnología, Universidad Nacional de entre Rios. Concepción del Uruguay, Argentina, XXXVI 50, 35-70.

El Editor; (2009). Rendimiento Académico en Cursos Universitarios. Formación Universitaria, R e c u p e r a d o d e : http://www.redalyc.org/articulo.oa?id=37354 0872006 [Consulta 04 de abril del 2016]

LECUYER, J. (2001). La evaluación en la enseñanza superior. Revista Diálogo Educacional, Pontificia Universidade católica do Paraná. Paraná, Brasil, 2(4), 1-26.

MORAL DE LA RUBIA, J. (2006). Predicción del rendimiento académico universitario. Perfiles Educativos. Instituto de Investigaciones sobre la Universidad y la Educación. Distrito Federal, México, XXVIII 113, 38-63.

PÁEZ M.; CASTAÑO \& CASTRILLÓN J. (2015). Inteligencia emocional y rendimiento académico en estudiantes universitarios. Psicología desde el Caribe, Universidad del Norte. Barranquilla, Colombia, 32, 268-285.

PÉREZ A. (2007). La evaluación como objeto de estudio. Avallacao: Revista de Avallacao da Educacao Superior. Universidade de Sorocoba. Sorocoba, 12(4), 583-596.

SUÁREZ L.; BARRIOS I. \& GONZALES M. (2007). Sistema de evaluación y acreditación de carreras universitarias. Implantación en el Instituto superior de ciencias Médicas de la Habana. Revista Habanera de ciencias Médicas, Universidad de ciencias Médicas de la Habana. Ciudad de la Habana. 6,1-11. 